appearance of cancer. Although the specificity for these responses is high, typically only $5-20 \%$ of patients demonstrate a response to any given antigen, which has limited the usefulness of single antigen responses as biomarkers. The recent development of protein microarrays may offer an ideal tool for screening for immune response to tumor antigens. These arrays offer the advantage that hundreds to thousands of different proteins can be printed and screened simultaneously and only require a few microliters of serum per assay.

Prof. LaBaer's group (Harvard Institute of Proteomics) has developed a novel method for producing protein microarrays called nucleic acid programmable protein arrays (NAPPA) that avoids the need to express and purify the proteins by substituting the printing of cDNAs on the arrays, which are then transcribed and translated in situ as needed at the time of the assay. NAPPA has been used successfully to map the pairwise protein interactions of the human DNA replication complex. Here, we propose adapting the NAPPA protein microarray technology for use in the rapid and efficient screening of sera from cancer patients for antibodies to 1000 known and potential tumor antigens in a multiplex format in order to better characterize the immune response to known tumor antigens, identify new informative tumor antigens and evaluate the value of using patterns of tumor antigen immune responses as biomarkers in Colorectal Cancer. For the validation of the possible biomarkers found in 20 different patients (pre- \& post-chemotherapy), currently we are using iFISH and SNPs approaches with the main goal to correlate genomics and functional proteomics.

\title{
Detection of Prostate Cancer by Urine Proteomics
}

\author{
Marina Rigau ${ }^{1}$, Núria Colome ${ }^{3}$, Juan Morote ${ }^{2}, M^{a}$ Carme Mir $^{2}$, Carlos Ballesteros ${ }^{2}$, Marta Garcia ${ }^{1}$, \\ Miguel Abal ${ }^{1}$, Francesc Canals ${ }^{3}$, Jaume Reventós ${ }^{1}$, Andreas Doll ${ }^{1}$
}

${ }^{1}$ Unitat de Recerca Biomèdica, Institut de Recerca. ${ }^{2}$ Servei d'Urologia. ${ }^{3}$ Laboratori de Proteòmica, Institut de Recerca. Hospital Vall d'Hebron, Barcelona

\section{Introduction}

Prostate-specific-antigen (PSA) serum measurement in combination with a digital rectal examination (DRE) and transrectal ultrasound-guided biopsy (TURS) is currently the gold standard for prostate cancer (PCa) screening in Europe [1]. Nevertheless, PSA and DRE lack significant specificity and biopsy lacks ideal sensitivity (12-30\% false negatives) [2, 3]. Therefore additional biomarkers are needed to supplement or potentially replace the currently used diagnostic techniques. As the secreted products from both, normal prostate epithelial cells as well as $\mathrm{PCa}$ cells, can be detected in the urine of men, their use as a proximal body fluid to detect $\mathrm{PCa}$ is very attractive since they could be the best compromise between a minimal invasive technique accepted by a wider range of the male population and the possibility to obtain enough cells for a correct diagnosis $[4,5]$.

\section{Objective}

We sought to determine a proteomic profile in urine able to distinguish between the presence and absence of PCa.

\section{Material\&Methods}

We used a combination of proteomic technologies in aged matched post-Digital Rectal Exam (DRE) urine supernatants specimens to identify differentially expressed proteins in patients with $\mathrm{PCa}$. Firstly, we depleted 9 histological confirmed $\mathrm{PCa}$ urine samples and 9 control samples (age-matched patients with the typical background of benign prostate hiperplasy (BPH), atrophy and chronic inflammation) using ProteoMiner (BioRad), a novel depletion technique that reduces the level of the most abundant species, while strongly concentrating the more dilute and rare ones. Then, Two-dimensional 


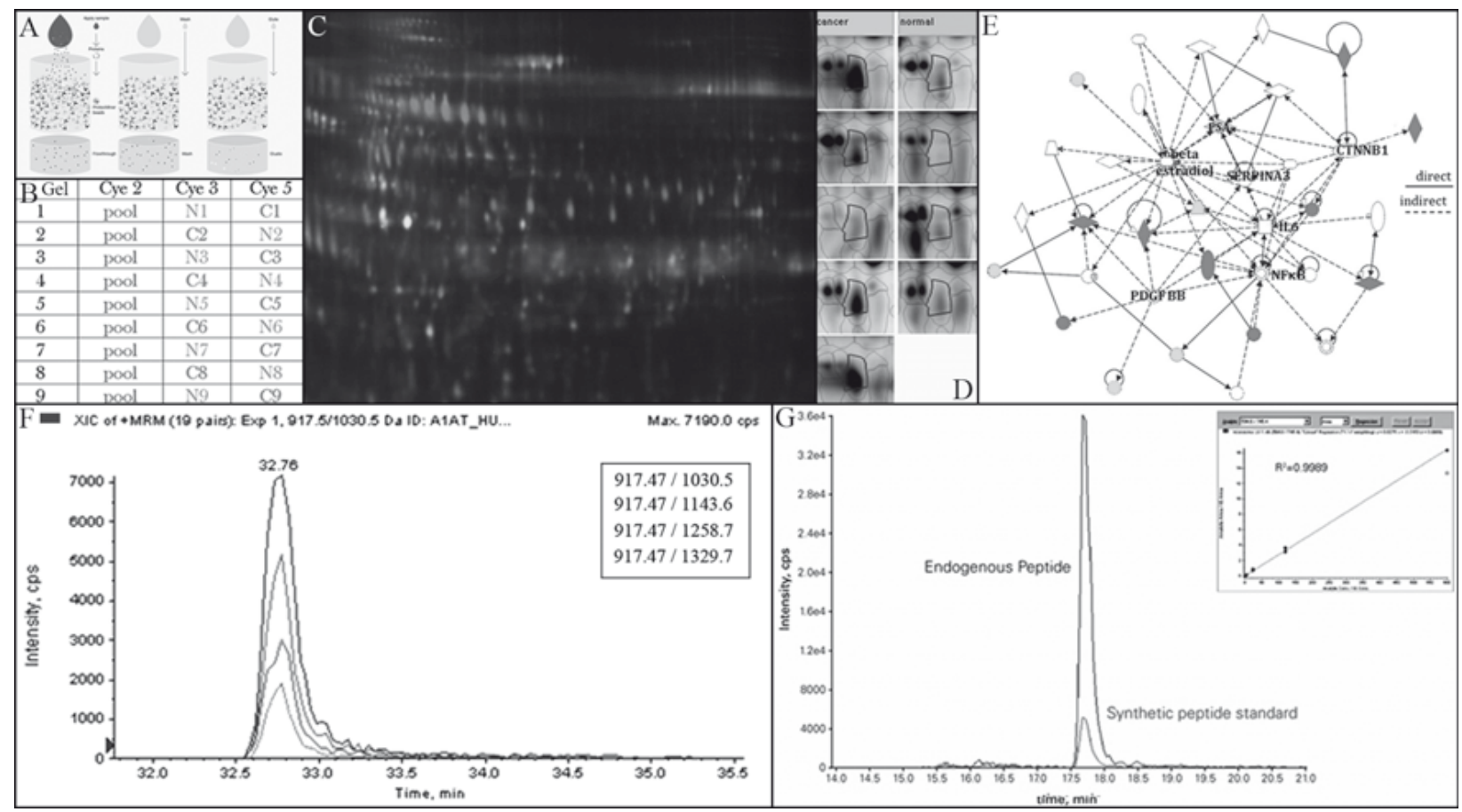

Figure 1. Protein identification by proteomic comparative analysis. (A) ProteoMiner depletion technique overview. (B) 2D-DIGE experiment design, comparison of 9 urine PCa samples against 9 urine control samples. (C) Example of a representive gel of the DIGE experiment. (D) Example of up-expressed spot in PCa samples. (E) IPA network interaction of over-expressed protein spots (red figures) and under-expressed protein spots (green figures). (F) MRM transitions of a tryptic peptide derived from one of the candidate biomarker proteins. (G) MRM-based absolute quantification of a peptide abundance using a labeled synthetic peptide as internal standard.

gel-based proteomic approach (2D-DIGE) coupled with matrix assisted laser desorption/ionization time-of-flight mass spectrometry (MALDI-TOF MS) and database mining experiment were performed to identify novel biomarkers of PCa in urine. Finally, we validated the candiadate biomarkers using Multiple Reaction Monitoring (MRM)-based assays, conducted by Liquid chromatography in conjunction with triple quadrupole mass spectrometry (LC-MS/ MS) in a bigger cohort of urine samples.

\section{Results}

We identified a proteomic profile of 26 potential biomarkers (16 down and 10 up-expressed) for the detection of $\mathrm{PCa}$ in urine. Ingenuity pathways analysis (IPA) showed that the majority of these proteins are secreted components of several well known functional of cancer and inflammation networks like NFKB, PDGFB $\beta$ and PSA. We performed MRM-based assays for 15 candidate biomarkers to quantify these in a sample set of 50 urine supernatants (Figure 1).

\section{Conclusions}

These data demonstrate the ability of proteomic analyses to reveal novel biomarkers for $\mathrm{PCa}$ in urine, an important step forward in advancing accurate diagnosis which is currently the bottleneck for the ability to cure patients from PCa. MRM is emerging as a technology that ideally complements the discovery capabilities of shotgun strategies by its unique potential for reliable quantification of analytes of low abundance in complex mixtures, such urine samples[6]. These biomarkers could be used to develop a a simple diagnostic test (probably similar to the ELISA, either multiplex or strips, reactive to the 4-5 molecules most representative of the differential profile) to be used in the hospital and outpatient routines.

\section{References}

[1] Catalona, W. J., Smith, D. S., Ratliff, T. L., Dodds, K. M., et al., Measurement of prostate-specific antigen in serum as a screening test for prostate cancer. N Engl J Med 1991; 324: 1156-1161. 
[2] Cervera Deval, J., Morales Olaya, F. J., Jornet Fayos, J., Gonzalez Anon, M., [Diagnostic value of the second prostate biopsies in males of risk. Study stratified by value of PSA]. Actas Urol Esp 2004; 28: 666-671.

[3] Raber, M., Scattoni, V., Salonia, A., Consonni, P., Rigatti, P., [Repeated ultrasound-guided transrectal prostate biopsy in patients with negative histologic test]. Arch Ital Urol Androl 2000; 72: 197-199.

[4] Okamoto, A., Yamamoto, H., Imai, A., Hatakeyama, S., et al., Protein profiling of post-prostatic massage urine specimens by surface-enhanced laser desorption/ionization time-of-flight mass spectrometry to discriminate between prostate cancer and benign lesions. Oncol Rep 2009; 21: 73-79.

[5] M'Koma, A. E., Blum, D. L., Norris, J. L., Koyama, T., et al., Detection of pre-neoplastic and neoplastic prostate disease by MALDI profiling of urine. Biochem Biophys Res Commun 2007; 353: 829-834.

[6] Lange, V., Picotti, P., Domon, B., Aebersold, R., Selected reaction monitoring for quantitative proteomics: a tutorial. Mol Syst Biol 2008; 4: 222.

\title{
NanoLC/mass spectrometry-based proteomic analysis of serum and synovial fluid samples from osteoarthritis patients
}

\author{
Patricia Fernández-Puente, Jesús Mateos, Carolina Fernández-Costa, Cristina Ruiz-Romero, \\ Francisco J Blanco
}

Osteoarticular and Aging Research Lab, Nodo Asociado de Proteo-Red. INIBIC-Hospitalario Universitario A Coruña, Xubias 84, 15006 - A Coruña, SPAIN

\section{Introduction}

Osteoarthritis (OA) is the most common rheumatic pathology, characterized mainly by cartilage degradation [1]. Despite its high prevalence, the diagnosis methods currently available are limited and lacked of sensitivity. Therefore, there is a considerable interest pointed in the characterization of new specific OA biomarkers in biological fluids. In this work we set up a nLC-MALDI-MS method for OA biomarker search in complex mixtures, with the aim of obtaining a standardized protocol for serum and synovial fluid (SF) protein profiling.

\section{Methods}

a) Sample preparation and immunodepletion: Serum and SF samples were obtained from OA patients and control donors. Prior to protein depletion, $\mathrm{SF}$ was treated with hyaluronidase. The 20 most abun- dant proteins were removed from crude serum and SF using the Immunodepletion column ProteoPrep ${ }^{\circledR} 20$ (Figure 1), according to manufacturer's instructions (Sigma Aldrich). Depletion of abundant proteins was checked by SDS-PAGE separation of the proteins and identification in a 4800 MALDI-TOF/TOF system (ABI). For both serum and SF depleted samples, protein concentration was determined using a nanoDrop instrument (Fisher Thermo Scientific, USA).

b) Pre-fractioning of the samples: For serum samples, digestion of the depleted fractions was done with trypsin, and the peptides obtained were fractionated by strong cation exchange (SCX) liquid chromatography in a HP 1200 system (Agilent), using a PolySulfoethyl column (PolyLC). SF proteins were separated by SDS-PAGE, using 10\% acrylamide gels. The gel lanes were divided into 12 sections, excised and proteins were in-gel digested with trypsin following standard procedures. 